\title{
EVANESCENT CORE PSEUDOPOTENTIAL: APPLICATIONS TO SURFACES AND CLUSTERS
}

\author{
C. FIOLHAIS, F. NOGUEIRA \\ and C. HENRIQUES* \\ Department of Physics, University of Coimbra, 3000 Coimbra, Portugal \\ *Department of Physics, FCTUNL, 2825 Monte da Caparica, Portugal
}

\begin{abstract}
A new local pseudopotential, called the "evanescent core" pseudopotential, has recently been proposed for sp-bonded metals. It is fitted to dominant density parameters of the solid state (valence, average equilibrium valence electron density, and interstitial valence electron density), and it yields an overall good description of physical properties such as binding energies and bulk moduli, in the framework of second-order perturbation theory. The potential, therefore, takes into account the atomic structure of the metal beyond the stabilized jellium model or structureless pseudopotential model. We present applications of the pseudopotential to surfaces and clusters of $\mathrm{Na}, \mathrm{Mg}$ and $\mathrm{Al}$, specifically: (i) Band-structure effects on surface tension and work functions; (ii) Cohesive energies and optimized structures of small clusters with two and six atoms (the latter with octahedral symmetry). The results are compared with those of the stabilized jellium model.
\end{abstract}

\section{Introduction}

Local pseudopotentials are very useful in solid state physics due to their simplicity and compatibility with Density Functional Theory [1, 2].

The "evanescent core" potential is a local pseudopotential constructed on the basis of second-order perturbation theory, assumed valid for simple metals. This potential is based on the concepts of smoothness and evanescence, i.e., exponential decay of the core repulsion at large distances [3, 4]. It is characterized by two parameters for each metal, which are adjusted to reproduce the experimental valence electron density parameter $r_{s}$ for a given valence $z$ (stability condition) and the theoretical average density in the interstitial region given by an all-electron calculation (interstitial density condition). One of these parameters, $R$, is related to the core size while the other, $\alpha$, fixes the single zero of the Fourier transform of the pseudopotential. In a 
new "universal" form the interstitial density is not needed, since the second parameter is fixed by minimizing the core radius $R$ over all the potentials satisfying the stability condition [5].

The bare evanescent core pseudopotential may be written as

$$
\omega(r)=-\frac{z}{R}\left\{\frac{1}{x}\left[1-(1+\beta x) e^{-\alpha x}\right]-A e^{-x}\right\},
$$

with $x=r / R$. The parameters $\beta$ and $A$ are functions of $\alpha$ determined by imposing vanishing first and third derivatives of the pseudopotential at the origin. This leads to a quick convergence in reciprocal space.

The stability condition is:

$$
\left.\frac{\partial e}{\partial r_{s}}\right|_{r_{s}=r_{s}^{\exp }}=0
$$

with

$$
e=e^{J}+e_{M}+\bar{\omega}_{R}+e_{B S},
$$

where $e^{J}$ is the binding energy per electron of the jellium model, $e_{M}$ is the Madelung energy, $\bar{\omega}_{R}$ is the average repulsive part of the pseudopotential and $e_{B S}$ is the band-structure energy, given by:

$$
e_{B S}=\frac{\bar{n}}{2 z^{2}} \sum_{\vec{G} \neq 0}|\omega(G) S(\vec{G})|^{2} \frac{\chi(G)}{\epsilon(G)}
$$

$\left(\bar{n}=\frac{3}{4 \pi r_{s}^{3}}\right.$ is the average electron density, $\omega(G)$ the Fourier transform of $\omega(r), S(\vec{G})$ the structure factor, $\chi(G)$ and $\epsilon(G)$, respectively, the response function and the dielectric function of Lindhard's screening theory with local-field exchange-correlation corrections in the Local Density Approximation, LDA).

On the other hand, the interstitial density condition is:

$$
N_{\text {int }}^{\text {unif }}-\left.4 \pi \frac{\bar{n}}{z} \sum_{\vec{G} \neq 0} \omega(G) \operatorname{Re}[S(\vec{G})] \frac{\chi(G)}{\epsilon(G) G^{3}}(\sin x-x \cos x)\right|_{x=G r_{m i n}}=N_{\text {int }}^{\text {all-electron }}
$$

with

$$
N_{\text {int }}^{\text {unif }}=z\left[1-\left(\frac{r_{\min }}{z^{1 / 3} r_{s}}\right)^{3}\right]
$$

the number of electrons in the interstitial region of an homogeneous system $\left(r_{\min }\right.$ is the radius of the inscribed sphere in the Wigner-Seitz cell).

The mechanical properties calculated with the potential (1) turned out to be in very good agreement with experiment [3]. They are generally better than non-perturbative results with nonlocal pseudopotentials, probably due to the stability condition (2). It was shown that nonlocality is unimportant for describing the energetics of most simple metals, including structural energy differences. On the contrary, some failures of the potential were clearly seen when its transferability to atoms was examined [4]. These were traced back to the imposed locality of the pseudopotential. Only in the case of sodium was that transferability almost perfect.

We examine here the transferability of the pseudopotential to surfaces and clusters of sodium, magnesium and aluminum. We restrict our attention to dimers, which are the smallest 
clusters, and six-atom clusters, which are the smallest clusters with a compact, three-dimensional shape. For surfaces we utilize a generalization of the stabilized jellium or structureless potential model [6] which incorporates band structure in a simple way, and do not attempt an atomistic approach. For clusters we do full atomistic calculations using a plane-wave solid state code [7], comparing the outcome with the results [4] obtained with a molecular code (Linear Combination of Atomic Orbitals, LCAO) [8] for the same systems. For surfaces as well as for clusters, and as we have done in [3] for the bulk material, we will compare the results with those of the stabilized jellium model.

\section{Surface results}

Stabilizea jellium [6] is a model which takes into account the lattice ions but still keeps the simplicity and universality of the well-known jellium model. One of its ingredients is the empty core Ashcroft pseudopotential, although any other local pseudopotential with a single parameter (as, e.g., the Shaw potential) would give the same results. In the stabilized jellium model the following energy density functional is used

$$
E_{S J}\left[n, n_{+}\right]=E_{J}\left[n, n_{+}\right]+\left(e_{M}+\bar{\omega}_{R}\right) \int d^{3} \vec{r} n_{+}(\vec{r})+\langle\delta v\rangle_{W S} \int d^{3} \vec{r} \frac{n_{+}(\vec{r})}{\bar{n}}\left[n(\vec{r})-n_{+}(\vec{r})\right],
$$

with $n=n(\vec{r})$ the valence electron density, $n_{+}$the constant positive background density, $E_{J}$ the jellium energy density functional (using the LDA for exchange and correlation), and $\langle\delta v\rangle_{W S}$ the average, in the Wigner-Seitz cell, of the difference between the pseudopotential and the jellium potential.

The surface properties of this model have been analyzed in Refs. $[6,9,10]$ using extended Thomas-Fermi as well as Lang-Kohn methods. However, in Ref. [6] a different density functional was proposed which is, a priori, superior, since it incorporates band structure effects in the constant potential $\langle\delta v\rangle_{W S}$ which is added perturbatively to the jellium potential:

$$
\begin{gathered}
E_{S J}^{\prime}\left[n, n_{+}\right]=E_{J}\left[n, n_{+}\right]+\left(e_{M}+\bar{\omega}_{R}+e_{B S}\right) \int d^{3} \vec{r} n_{+}(\vec{r})+ \\
+\left(\langle\delta v\rangle_{W S}+\mu_{B S}\right) \int d^{3} \vec{r} \frac{n_{+}(\vec{r})}{\bar{n}}\left[n(\vec{r})-n_{+}(\vec{r})\right],
\end{gathered}
$$

with $e_{B S}$ and $\mu_{B S}$ the band-structure contributions to the energy and chemical potential.

It is not appropriate to use the Ashcroft potential in the functional (8), since it yields too big band-structure energies and potentials if the sums in reciprocal space are correctly done. Ashcroft and Langreth [11] were only able to reproduce the mechanical properties of metals by decoupling the $\vec{G}=0$ from the $\vec{G} \neq 0$ components of the Ashcroft potential and adjusting a parameter in the $\vec{G}=0$ term using the stability condition at the observed density. (Moreover, they have considered a single shell in reciprocal space). We may however use a pseudopotential, such as the evanescent core, with good convergence properties in the reciprocal space. 


\begin{tabular}{||c||cc||c|c|c|c||}
\hline Metal & $r_{s}$ & $z$ & $\langle\delta v\rangle_{W S}^{S J}$ & $\mu_{B S}$ & $\langle\delta v\rangle_{W S}^{S J}+\mu_{B S}$ & $\langle\delta v\rangle_{W S}^{S J}$ \\
\hline \hline $\mathrm{Na}$ & 3.93 & 1 & +0.150 & -0.161 & -0.011 & -0.088 \\
\hline $\mathrm{Mg}$ & 2.65 & 2 & -0.511 & -0.420 & -0.931 & -1.113 \\
\hline $\mathrm{Al}$ & 2.07 & 3 & -1.695 & -0.885 & -2.581 & -2.491 \\
\hline
\end{tabular}

\begin{tabular}{||c||c|c|c||c|c|c||}
\hline Metal & $W^{S J^{\prime}}$ & $W^{S J}$ & $W^{\text {exp }}$ & $\sigma^{S J}$ & $\sigma^{S J}$ & $\sigma^{\text {exp }}$ \\
\hline \hline $\mathrm{Na}$ & 2.85 & 2.86 & 2.75 & 170 & 179 & 218 \\
\hline $\mathrm{Mg}$ & 3.73 & 3.77 & 3.66 & 476 & 533 & 654 \\
\hline $\mathrm{Al}$ & 4.28 & 4.26 & 4.28 & 979 & 928 & 953 \\
\hline
\end{tabular}

Table 1: Above: Constant potential of the stabilized jellium model in the versions given, respectively, by the functionals (7) and (8) (labelled by $S J$ and $S J^{\prime}$ ). $r_{s}$ in bohr and the other quantities in $\mathrm{eV}$. Below: Work function $W$ and surface tension $\sigma$ obtained in the stabilized jellium model given by functionals (7) and (8). The experimental values are from Ref. [9]. $W$ in $\mathrm{eV}$ and $\sigma$ in $\mathrm{erg} / \mathrm{cm}^{2}$.

Table 1 shows the values of the constant potential $\langle\delta v\rangle_{W S}$ obtained in the framework of the energy functionals (7) and (8), and the corresponding results for the surface properties from a Lang-Kohn surface calculation. The values of the work function and surface tension with and without band-structure effects are close. The work functions obtained with the functional (8) are marginally better in comparison with experiment than those arising from functional (7). The same is true for the surface tension of $\mathrm{Al}$, but not for the surface tensions of $\mathrm{Mg}$ and $\mathrm{Na}$. Our results of Table 1 labelled with $S J$ differ a little from those of Ref. [9] since we have improved the input of the self-consistent equations for each particular metal.

\section{Cluster results}

Clusters are systems intermediate between bulk matter and free atoms, and represent a potentially useful application of our local pseudopotential based on solid state properties. It has been shown $[9,12,13]$ that surface and curvature energies suffice to determine the "smooth" (non-shellstructure) part of the energy of small clusters of jellium or stabilized jellium. The evanescent core pseudopotential has already been used to determine the nearest-neighbour distances and cohesive energies of $\mathrm{Na}, \mathrm{Mg}$ and $\mathrm{Al}$ dimers and octahedral clusters [4]. The calculation of [4] employed a linear-combination-of-atomic-orbitals local density molecular code [8]. Here we investigate further the energetics of two and six-atom clusters, with the same ionic geometries but a different method. No attempt has been made to optimize the geometry of the six-atom clusters. At the end, we contrast the results with those of the stabilized jellium model.

We have solved the Kohn-Sham equations in the LDA using the Perdew-Zunger parametrization of the Ceperley-Alder results for correlation with the clusters placed in a large supercell with periodic boundary conditions. The size of the supercells was chosen large enough to 
render negligible the interaction between a cluster and its periodic images. For the dimers a bcc supercell with side 40 bohr was used, while for the octahedral clusters a sc supercell with side 32 bohr was used. A plane wave expansion was used to sample the Brillouin zone of the supercells. That expansion was made around the $\Gamma$ point with an energy cutoff of 12 Ry. Our results are summarized in Tables 2 and 3 .

Cohesive energy $\epsilon_{c}$ Density parameter $r_{s}^{*}$

\begin{tabular}{||c|c|c|c|c|||c|c|c|c|c||}
\hline Cluster & PW & LCAO & All-electron & Exp. & Cluster & PW & LCAO & All-electron & Exp. \\
\hline \hline $\mathrm{Na}_{2}$ & 0.59 & 0.46 & 0.44 & 0.37 & $\mathrm{Na}_{2}$ & 3.16 & 3.19 & 3.12 & 3.22 \\
\hline $\mathrm{Mg}_{2}$ & 0.06 & 0.04 & 0.09 & 0.03 & $\mathrm{Mg}_{2}$ & 3.07 & 3.15 & 2.85 & 3.22 \\
\hline $\mathrm{Al}_{2}$ & 0.51 & - & - & 0.78 & $\mathrm{Al}_{2}$ & 2.03 & - & - & 1.96 \\
\hline
\end{tabular}

Table 2: Left: Cohesive energies for dimers, in $\mathrm{eV} /$ atom. $\mathrm{PW}$ is the present calculation, using the LDA for the cluster and the atom. LCAO calculations employed Local Spin Density (LSD) for the atom, and LDA for the cluster [4]. The all-electron results, obtained also in a LCAO approach, employed LSD for both [14]. Right: Average valence electron density parameters $r_{s}^{*}$ for dimers. $r_{s}^{*}=0.5527 b / z^{1 / 3}$, where $b$ is the nearest-neighbour bond length. The experimental values are from Refs. [15], [16], and [17].

For the sodium clusters considered we found bond lengths that agree well with those found previously with the same or other potentials $[4,18]$. Our result for the dimer bond length ( $5.71 \mathrm{bohr}$ ) is somewhat closer to the experimental value ( $5.82 \mathrm{bohr}$ ) [15] than the result ( $5.5 \mathrm{bohr}$ ) of Ref. [18], in which the interatomic distances were underestimated. Our dimer cohesive energy is bigger than the experimental value. For the dimer we found the vibrational frequency to be $148 \mathrm{~cm}^{-1}$, which compares very well with the experimental value $159 \mathrm{~cm}^{-1}$ [15]. For the $\mathrm{Na}_{6}$ cluster we found a bond length ( 6.32 bohr) which is bigger than those of the flattened pentagonal pyramid which is the proposed minimal energy structure of Ref. [18].

Cohesive energy $\epsilon_{c}$

Density parameter $r_{s}^{*}$

\begin{tabular}{||c|c|c|c|||c|c|c|c||}
\hline Cluster & PW & LCAO & All-electron & Cluster & PW & LCAO & All-electron \\
\hline \hline $\mathrm{Na}_{6}$ & 0.67 & 0.53 & 0.61 & $\mathrm{Na}_{6}$ & 3.49 & 3.80 & 3.60 \\
\hline $\mathrm{Mg}_{6}$ & 0.21 & 0.17 & 0.23 & $\mathrm{Mg}_{6}$ & 3.09 & 2.98 & 2.80 \\
\hline $\mathrm{Al}_{6}$ & - & 2.02 & 2.12 & $\mathrm{Al}_{6}$ & - & 2.01 & 2.01 \\
\hline
\end{tabular}

Table 3: Same as Table 2 for octahedral clusters.

For $\mathrm{Mg}_{2}$ the agreement with experiment of the cohesive energy and the bond length, which is better than in other calculations [19], is somewhat surprising. Kumar and Car [19] have studied three different structures of $\mathrm{Mg}_{6}$. One of them is a distorted octahedron with bond lengths close to their dimer bond length, as we have also found.

For $\mathrm{Al}_{2}$ our bond length is in good agreement with the experimental value [17], but our vibrational frequency $\left(185 \mathrm{~cm}^{-1}\right)$ is $35 \%$ smaller than the experimental value $\left(284 \mathrm{~cm}^{-1}\right)$ [17]. 
For the octahedral $\mathrm{Al}_{6}$ cluster, we did not obtain a convergent self-consistent solution with the plane-wave method.

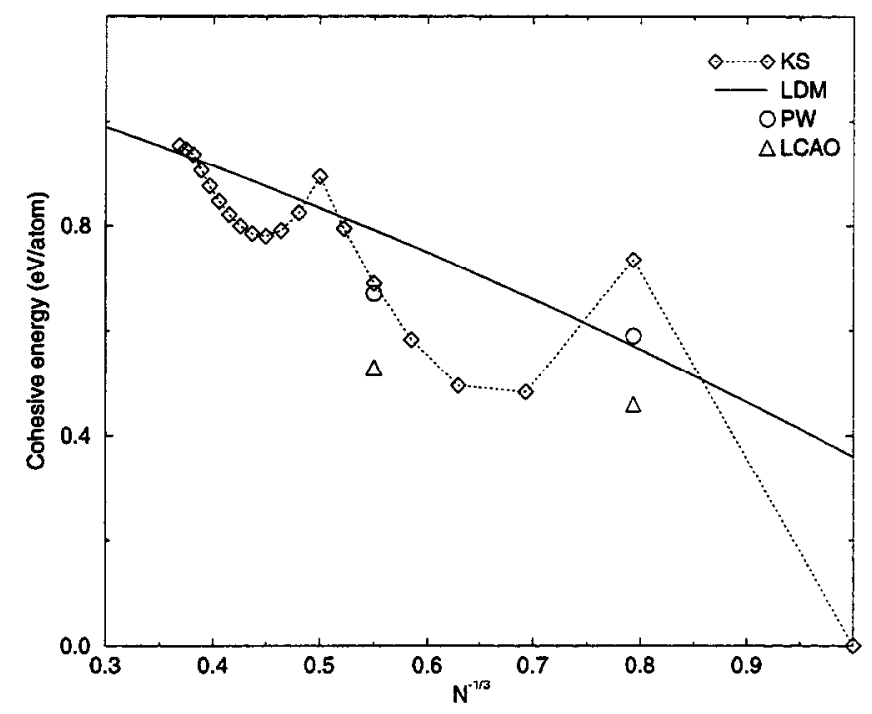

Figure 1: Comparison of the cohesive energies of $\mathrm{Na}_{2}$ and $\mathrm{Na}_{6}$ obtained with the plane-wave (PW) and the linear-combination-of-atomic-orbitals (LCAO) methods with the stabilized jellium results (Kohn-Sham, KS, and Liquid-Drop-Model, LDM). $\mathrm{N}$ is the number of atoms in the cluster.

Figs. 1-3 compare the cohesive energies of $\mathrm{Na}, \mathrm{Mg}$ and $\mathrm{Al}$ two- and six-atom clusters determined with the plane-wave method and the LCAO against spherical stabilized jellium results (Kohn-Sham and Liquid-Drop-Model, including surface and curvature energies, based on functional (7)). In the stabilized jellium model, the self-compression effect was not considered. The atomistic values are lower than those of the continuous model, except for $\mathrm{Mg}$.

\section{Conclusions}

We have shown that a modification of the stabilized jellium model which incorporates band-structure effects obtained with the evanescent core potential leads to work functions which are slightly better than those of the standard stabilized jellium model. However, there is no such improvement for the surface tension.

We have also shown that cluster calculations done with a plane-wave code are close to those found previously with a molecular code using the same pseudopotential. The bond lengths from the plane-wave code tend to be a little smaller, in better agreement with all-electron results. An interesting result is that octahedral $\mathrm{Na}_{6}$ and $\mathrm{Al}_{6}$ clusters are found to be compressed with respect 


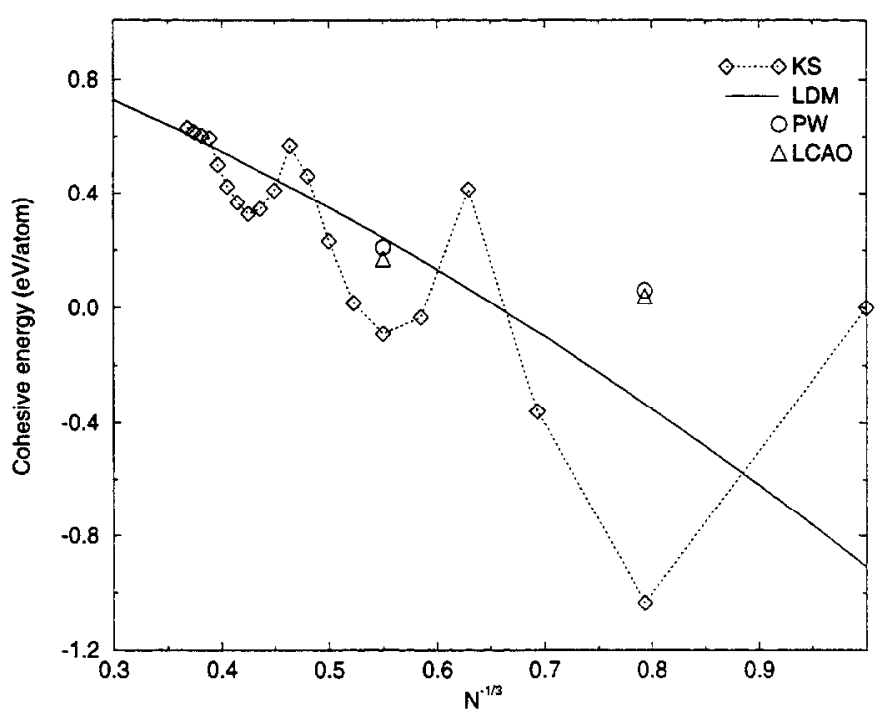

Figure 2: Same as Fig. 1, for magnesium clusters.

to the bulk metal while $\mathrm{Mg}_{6}$ expands $[4,14]$. The stabilized jellium model predicts a similar compression for metal clusters due to the surface tension [20, 21]. The expansion found for $\mathrm{Mg}_{6}$ contrasts with the compression predicted by the stabilized jellium model. To explain this we note that, with increasing cluster size, $\mathrm{Mg}$ is expected to pass over from van der Waals to metallic binding [14, 22, 23]. If $\mathrm{Mg}_{6}$ is much less metallic than bulk $\mathrm{Mg}$, its weakened bond could give rise to an expansion of the bond length.

We have used potentials fitted to bulk properties in second-order perturbation theory. For the cluster calculations, it would be more correct to re-fit the pseudopotential parameters in a non-perturbative calculation for the bulk. This would lead to smaller bond lengths, as already noted for bulk $\mathrm{Na}[4]$.

\section{Acknowledgements}

We thank A. Vieira for help in producing Figs. 1-3. We thank J. P. Perdew and J. L. Martins for helpful discussions. This work has been partially supported by the Portuguese PRAXIS XXI program (PRAXIS/2/2.1/FIS/26/94).

\section{References}

[1] W. A. Harrison, Pseudopotentials in the Theory of Metals, Benjamin, New York (1966). 


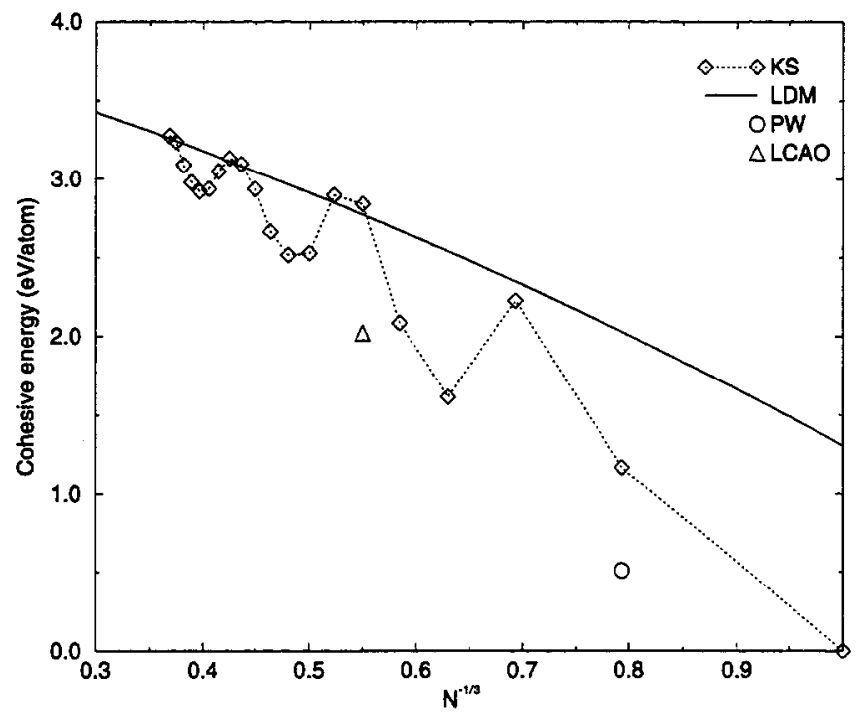

Figure 3: Same as Fig. 1, for aluminum clusters.

[2] W. E. Pickett, Comp. Phys. Rep. 92, 45 (1994).

[3] C. Fiolhais, J. P. Perdew, S. Q. Armster, J. MacLaren, and M. Brajczewska, Phys. Rev. B 51, 14001 (1995); Erratum: Phys. Rev. B 53, 13193 (1996).

[4] F. Nogueira, C. Fiolhais, J. He, J. P. Perdew, and A. Rubio, J. Phys.: Condens. Matter 8, 287 (1996).

[5] F. Nogueira, C. Fiolhais, and J. P. Perdew, in preparation.

6] J. P. Perdew, H. Q. Tran, and E. D. Smith, Phys. Rev. B 42, 11627 (1990).

77] J. Ihm, A. Zunger, and M. L. Cohen, J. Phys. C: Solid State Phys. 12, 4409 (1979).

[8] W. Yang, Phys. Rev. A 44, 7823 (1991).

[9] C. Fiolhais and J. P. Perdew, Phys. Rev. B 45, 6207 (1992).

[10] A. Kiejna, Phys. Rev. B 47,7361 (1993).

[11] N. W. Ashcroft and D. C. Langreth, Phys. Rev. 155, 682 (1967).

12] M. Brajczewska, C. Fiolhais, and J. P. Perdew, Int. J. Quantum Chem. S 27, 249 (1993).

[13] P. Ziesche, J. P. Perdew, and C. Fiolhais, Phys. Rev. B 49, 7916 (1994); Phys. Rev. B 50, 5020 (1994).

[14] G. S. Painter and F. W. Averill, Phys. Rev. B 50, 5545 (1994).

[15] K. K. Verma, J. T. Bahns, A. R. Rejei-Rizi, W. C. Stwalley, and W. T. Zemke, J. Chem. Phys. 78, 3599 (1983).

[16] K. P. Huber and G. Herzberg, Molecular Spectra and Molecular Structure. IV. Constants of Diatomic Molecules, Van Norstrand Reinhold, New York (1974).

[17] M. F. Cai, T. P. Djugan, and V. E. Bondybey, Chem. Rev. Lett. 155, 430 (1989).

[18] J. L. Martins, J. Buttet, and R. Car, Phys. Rev. B 31, 1804 (1985).

[19] V. Kumar and R. Car, Phys. Rev. B 44, 8243 (1991).

20] J. P. Perdew, M. Brajczewska, and C. Fiolhais, Solid State Commun. 88, 795 (1993).

[21] M. Brajczewska, C. Fiolhais, A. Vieira, and J. P. Perdew, in Proceedings of the International Conference on Many-Body Physics, Coimbra, Portugal, 20-25 September 1993, C. Fiolhais, M. Fiolhais, C. Sousa, and J. N. Urbano (Eds.), World Scientific, Singapore (1994).

[22] X. G. Gong, Q. Q. Zheng, and Yi-zhen He, Phys. Lett. A 181, 459 (1993).

[23] G. M. Pastor and K. H. Bennemann, in Clusters of Atoms and Molecules, H. Haberland, (Ed.), Springer, Berlin (1994). 